\title{
Construction and Application of Effective Path Statistics Network
}

\author{
Gang Zhang ${ }^{1}$,Qing-Kui Chen ${ }^{1,2}$ \\ (1.University of Shanghai for Science and Technology, Business School, \\ 20093, China) \\ (2.University of Shanghai for Science and Technology, School of Optical- \\ Electrical and Computer Engineer, 200093, China) \\ E-mail
}

\begin{abstract}
The Internet has dynamic change nature and the resources limitation that cause the loss of some commands, and thus the failure of group command transmission in the course of transmitting. On account of this, the paper presents Effective Path Statistics Network (EPSN), converting the group command transmission based on the Internet into the one based on EPSN. It describes the definition, construction and working principle of EPSN, and further conducts sampling and analyzing of metrics according to it. Based on the result the paper illustrates modeling and calculating of group command transmission. The experiments show that EPSN can effectively relieve the negative effect caused by the Internet dynamic change nature and the resources limitation.
\end{abstract}

Keywords: We would like to encourage you to list your keywords in this section.

\section{Introduction}

The Operation Center of the Internet of Things is the focus of the present research of the Internet of Things (IOT). Its main function is to transmit a group of commands of multi-constraints on the Internet and at the same time enable the whole command group to reach the intelligent terminals distributed in the different large areas (Large area refers to the one across provinces, cities and districts). In the course of group command transmission, the loss of any single command is regarded as group command transmission failure.

From the above we know that the nature of operation center can be regarded as Group Command Transmission Problem (GCTP) on the basis of the Internet.

The research on the GCTP based on the Internet is relatively rare. Theoretically, literatures [1] and [2] respectively explain the research significance of GCTP, point out that it is the key core of constructing intelligent internet of things, and further put forward some suggestions and thoughts to solve GCTP - from the angle of theory only. Technically, the present transmission technology mainly includes Unicast Routing ${ }^{[3-4]}$, Multicast Routing ${ }^{[5]}$, K-shortest Path $^{[6-7]}$ and $\mathrm{CMT}^{[8]}$ (Concurrent Multipath Transfer). But the existing technology is different from GCTP in terms of transmission means, so it is not applicable for GCTP.

Therefore, the research of GCTP based on the Internet is significant both theoretically and technically.

But the Internet has dynamic nature and resources limitation, difficulties may occur in the course of supporting group command transmission based on the service mode of trying best in the terms of two aspects. One is the liability of path expiration and 
invalidity under the dynamic environment. The other is path competition in the condition of resources limitation.

In view of this, this paper probes into the configuration of some Communication Service Agents (CSA) on the Internet on the basis of overlay network thought, constructs backbone network related to the Internet local area of operation center by means of undirected graph via CSA, i.e. Effective Path Statistics Network (EPSN) showed in diagram 1, EPSN topology.

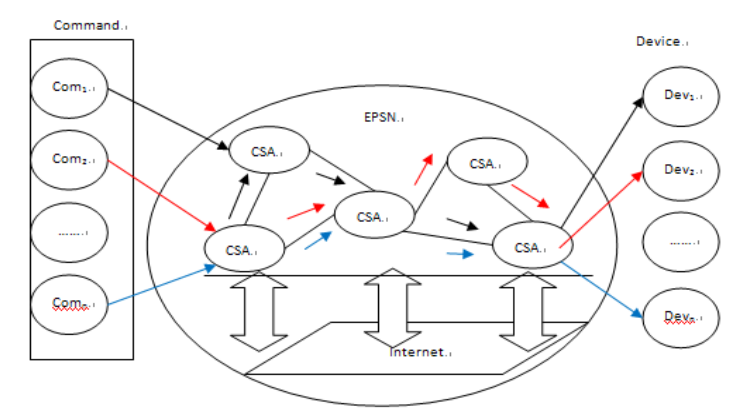

Diagram 1: EPSN Topology

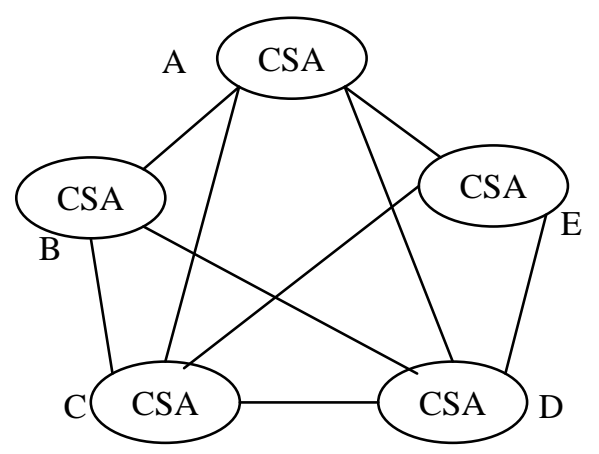

Diagram 2: Part of EPSN Topology

Diagram 1, $\operatorname{Com}_{i}, \operatorname{Dev}_{\mathrm{i}}(\mathrm{i}=1,2, \ldots, \mathrm{n})$ respectively represents command and terminal equipment while CSA represents communication service agent. Diagram 1 can be described as EP set constructed on the basis of EPSN, the operation center chooses a group of effective path EP set for group command $\mathrm{Com}_{1}, \mathrm{Com}_{2}, \mathrm{Com}_{\mathrm{n}}$, so that the group command can be transmitted to different intelligent terminals.

The significance of net construction lies in the real-time measurement and statistics of the present situation of the Internet via EPSN and thus reducing the ineffectiveness of the path caused by the dynamic nature of the Internet, and also the digging out of more effectively "void" resources, by means of which the group command transmission can be better conducted.

In summary, the problem under discussion in the paper can be converted to group command transmission problem (GCTP) based on EPSN.

The paper mainly presents EPSN, converts GCTP on the Internet to the one on EPSN, describes the definition, construction and working principle of EPSN, and further conducts sampling and analyzing of metrics according to EPSN. Based on the result the paper illustrates modeling and calculating of GCTP. The experiments show that EPSN can effectively solve GCTP. 


\section{EPSN STRUCTURE}

\subsection{EPSN Definition}

EPSN (Effective Path Statistics Network, EPSN) is a simple, practical, abstract and virtual network. It is a network logically linked via undirected graph by means of the deployment of CSA on the Internet. Each CSA node with simple function is only composed of transmitting module, receiving module and interactive storage module. EPSN mainly functions via CSA in the way of transmitting to each other, receiving and storing of the parameter information on the logical link, hence the interactive storage and statistics of parameter information among CSA. Finally, it constructs the real-time effective path (EP) set according to the statistics.

\subsection{EPSN Construction}

Topology is produced at random via Waxmam ${ }^{[9]}$ according to the completely random node deployment thought. All the CSA nodes deployed on the Internet are logically linked via undirected graph according to net topology, and each CSA node degree is between 2 and 7 .

\subsection{EPSN Working Thought}

EPSN divides every day within the whole year of 365 days into peak period, idle period and stable period. It continuously within 24 hours measures and does statistical work for the metrics information on the logical link between every two CSA nodes (This paper only discusses delay and delay jitter with the supposition that the logical link between every two CSA nodes and each sample parameter are independent of each other.). The sampling is done with the interval of five minutes. If the time span is exceeded, the restart time is 30 seconds. Finally, the EP set is constructed according to measuring and statistical result.

\subsection{EPSN Sampling Data Analysis}

Diagram 2 describes part of net topology constructed under the circumstance of the Internet and composed of five CSA nodes in Shanghai, China. The downtown area includes Yangpu District, Pudong District, Songjiang District, Minhang District and Jiading District. Each CSA degree is between 2 and 7. (To facilitate the distinction between different regions' CSA node, the districts are respectively marked as $\mathrm{A}, \mathrm{B}, \mathrm{C}, \mathrm{D}$ and $\mathrm{E}$ )

The following data under discussion was got between December, 2013 and March, 2014 on the basis of the result from the measuring and statistical work of net topology seen in diagram 2 above. (This paper only discusses delay and delay jitter.)

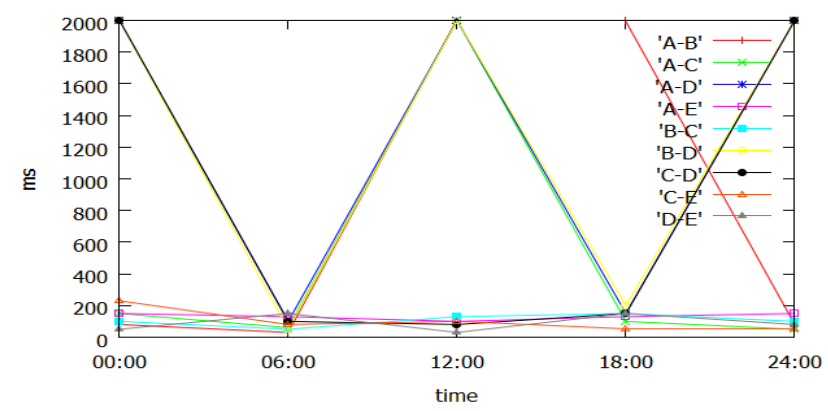

Diagram 3: Monthly Average Delay 
Diagram 3 discusses monthly average delay. On the abscissa are the peak period, idle period and stable period while the ordinate represents delay (ms). During the 0:00 period in the morning, the average delay of Link A-D, B-D and C-D has already exceeded $2,000 \mathrm{~ms}$, the average delay of link A-B is $80 \mathrm{~ms}$ while A-C and A-E $150 \mathrm{~ms}$. The average delays of link B-C, C-E and D-E are $100 \mathrm{~ms}, 230 \mathrm{~ms}$ and $50 \mathrm{~ms}$ respectively. During the 6:00 period in the morning, the average delays of Link A-B and $A-C$ are $30 \mathrm{~ms}$ and $60 \mathrm{~ms}$ while A-D and C-D $100 \mathrm{~ms}$. The average delay of A-E is $130 \mathrm{~ms}$ while B-C and B-D 50ms. The average delays of C-E and D-E are $80 \mathrm{~ms}$ and $150 \mathrm{~ms}$. During the 12:00 period at noon, the average delay of A-B, A-C, A-D and B$\mathrm{D}$ is $2000 \mathrm{~ms}$ while A-E and C-E $100 \mathrm{~ms}$. The average delays of B-C, C-D and D-E are respectively $130 \mathrm{~ms}, 80 \mathrm{~ms}$ and $30 \mathrm{~ms}$. During the $18: 00$ period in the afternoon, the average delays of A-B and A-C are $2000 \mathrm{~ms}$ and $100 \mathrm{~ms}$ while A-D, B-C, C-D and D-E $150 \mathrm{~ms}$. The average delays of A-E, B-D and C-E are $130 \mathrm{~ms}, 200 \mathrm{~ms}$ and $50 \mathrm{~ms}$ respectively. During the 24:00 period in the morning, the average delay of A-B and D$\mathrm{E}$ is $80 \mathrm{~ms}$ while A-C and C-E 50ms. The average delay of A-D B-D and C-D is $2000 \mathrm{~ms}$ while A-E and B-C $150 \mathrm{~ms}$ and $100 \mathrm{~ms}$.

To sum up, during the 6:00 period in the morning and 18:00 in the afternoon, the net is in idle and stable period while during the period of 00:00 in the morning, 12:00 at noon and 24:00 in the morning, the net is in peak period.

Table 1 Monthly Average Delay Jitter

\begin{tabular}{|c|c|c|c|c|c|}
\hline me & 00:00 & 06:00 & 12:00 & 18:00 & 24:00 \\
\hline A-B & {$[-80,80]$} & {$[-50,50]$} & $\begin{array}{c}{[-} \\
5000,5000] \\
\end{array}$ & $\begin{array}{c}{[-} \\
5000,5000] \\
\end{array}$ & {$[-80,80]$} \\
\hline A-C & {$[-200,200]$} & {$[-60,60]$} & $\begin{array}{c}{[-} \\
100,100] \\
\end{array}$ & {$[-80,80]$} & {$[-30,30]$} \\
\hline A-D & {$[-50,50]$} & $\begin{array}{c}{[-} \\
5000,5000] \\
\end{array}$ & $\begin{array}{c}{[-} \\
5000,5000] \\
\end{array}$ & {$[-300,300]$} & {$[-5000,5000]$} \\
\hline A-E & {$[-100,100]$} & {$[-90,90]$} & {$[-80,80]$} & $\begin{array}{c}{[-} \\
5000,5000]\end{array}$ & {$[-90,90]$} \\
\hline B-C & {$[-5000,5000]$} & $\begin{array}{c}{[-} \\
5000,5000] \\
\end{array}$ & $\begin{array}{c}{[-} \\
5000,5000] \\
\end{array}$ & {$[-200,200]$} & {$[-5000,5000]$} \\
\hline B-D & {$[-5000,5000]$} & $\begin{array}{c}{[-} \\
5000,5000]\end{array}$ & $\begin{array}{c}{[-} \\
400,400]\end{array}$ & {$[-300,300]$} & {$[-5000,5000]$} \\
\hline C-D & {$[-2000,2000]$} & $\begin{array}{c}{[-} \\
1000,1000] \\
\end{array}$ & $\begin{array}{c}{[-} \\
500,500] \\
\end{array}$ & {$[-200,200]$} & {$[-5000,5000]$} \\
\hline C-E & {$[-150,150]$} & {$[-60,60]$} & $\begin{array}{c}{[-} \\
500,500] \\
\end{array}$ & {$[-80,80]$} & {$[-50,50]$} \\
\hline D-E & {$[-600,600]$} & $\begin{array}{c}{[-} \\
100,100]\end{array}$ & $\begin{array}{c}{[-} \\
300,300]\end{array}$ & {$[-150,150]$} & {$[-100,100]$} \\
\hline
\end{tabular}

Table 1 discusses monthly average delay jitter. During the period of 00:00 in the morning, the average delay jitter of link B-C and B-D is [-5000, 5000]ms while C-D $[-2000,2000] \mathrm{ms}, \mathrm{D}-\mathrm{E}[-600,600] \mathrm{ms}$, all the others are below [-200, 200]ms. During the period of 06:00 in the morning, the average delay jitter of A-D, B-C and B-D is [$5000,5000] \mathrm{ms}$ while C-D [-1000,1000]ms, all the others are below [-200, 200]ms. During the period of 12:00 at noon, the average delay jitter of A-B, A-D and B-C is [5000, 5000]ms, while C-D and C-E [-500, 500]ms, D-E [-300, 300]ms, B-D [-400, $400] \mathrm{ms}$, all the others are below [-200, 200]ms. During the period of 18:00 in the afternoon, the average delay jitter of the link A-B and A-E is [-5000, 5000]ms while A-D and B-D [-300, 300]ms, all the others are below [-200, 200]ms. During the 
period of 24:00, the average delay jitter of A-D, B-C, B-D and C-D is [-5000, $5000] \mathrm{ms}$, all the others are below [-200, 200]ms.

To sum up, during the period of 18:00 in the afternoon, the net is in stable period while 00:00 and 06:00 in the morning, 12:00 at noon and 24:00 in the morning peak period.

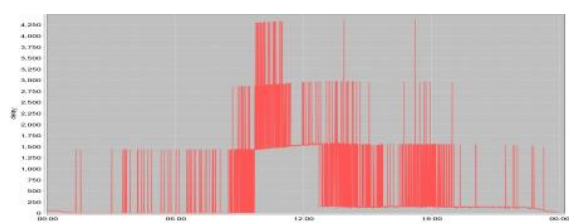

(A) A-B Averäge Delay

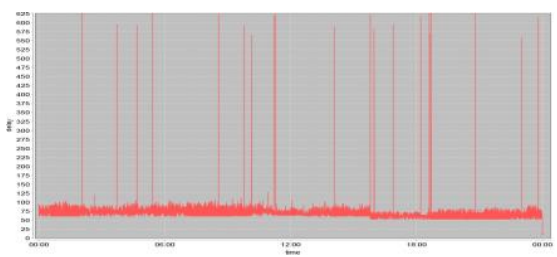

(C) A-E Average Delay

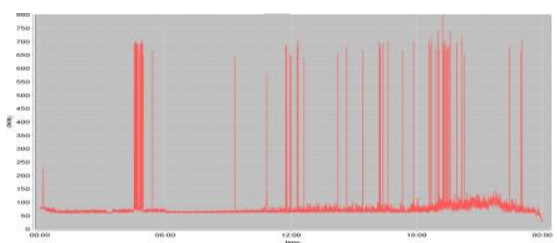

(E) B-C Average Delay

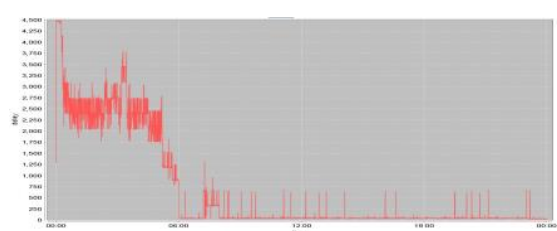

(G) C-D Average Delay

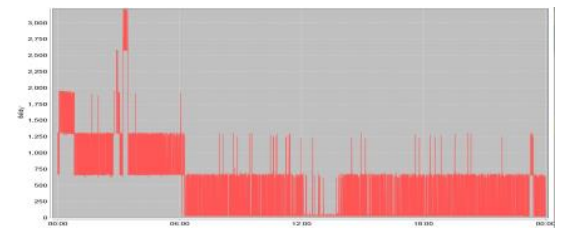

(B) A-C Average Delay

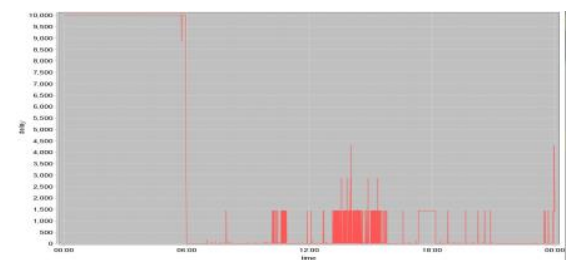

(D) A-D Average Delay

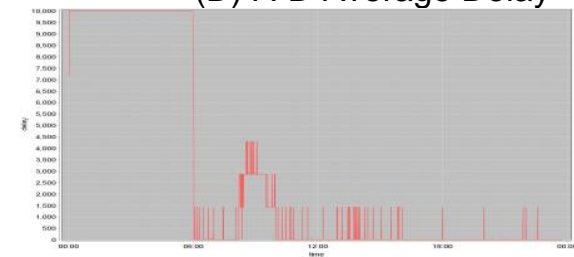

(F) B-D Average Delay

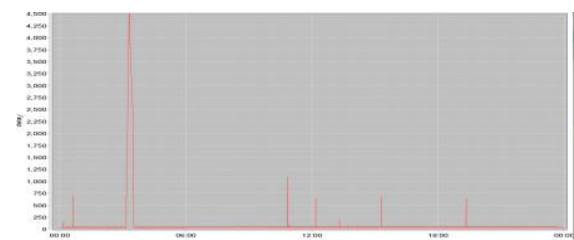

(H) C-E Average Delay

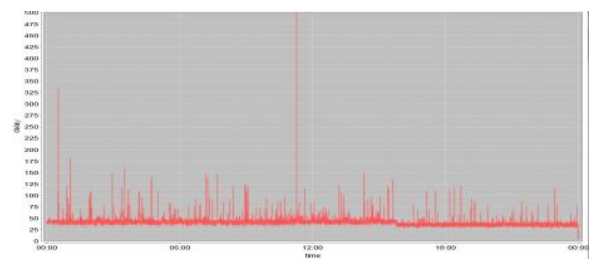

(I) D-E Average Delay

\section{Diagram 4: Daily Average Delay}

Diagram 4 (a) - (i) discusses daily average delay randomly selected between December, 2013 and March, 2014. The abscissa shows peak period, idle period and stable period, while the ordinate delay (ms). During the period of 00:00 in the morning, the link of A-B and B-C is $80 \mathrm{~ms}$ while A-C, A-E, A-D, B-D, C-D, C-E and D-E $700 \mathrm{~ms}, 700 \mathrm{~ms}, 5000 \mathrm{~ms}, 5000 \mathrm{~ms}, 1200 \mathrm{~ms}, 50 \mathrm{~ms}$ and $40 \mathrm{~ms}$ respectively. During the period of 06:00 in the morning the average delays of A-B, A-C, A-E, A-D, B-C, B-D, C-D, C-E and D-E are $1300 \mathrm{~ms}, 1000 \mathrm{~ms}, 75 \mathrm{~ms}, 100 \mathrm{~ms}, 70 \mathrm{~ms}, 1500 \mathrm{~ms}, 80 \mathrm{~ms}$, $60 \mathrm{~ms}$ and $45 \mathrm{~ms}$ respectively. During the period of 12:00 at noon the average delays of A-B, A-C, A-E, A-D, B-C, B-D, C-D, C-E and D-E are $1500 \mathrm{~ms}, 300 \mathrm{~ms}, 70 \mathrm{~ms}, 80 \mathrm{~ms}$, $70 \mathrm{~ms}, 100 \mathrm{~ms}, 80 \mathrm{~ms}, 60 \mathrm{~ms}$ and $60 \mathrm{~ms}$ respectively. During the period of 18:00 in the afternoon, the average delays of A-B, A-C, A-E, A-D, B-C, B-D, C-D, C-E and D-E are $800 \mathrm{~ms}, 300 \mathrm{~ms}, 70 \mathrm{~ms}, 1500 \mathrm{~ms}, 80 \mathrm{~ms}, 100 \mathrm{~ms}, 80 \mathrm{~ms}, 50 \mathrm{~ms}$ and $45 \mathrm{~ms}$ respectively. 
During the period of 24:00 in the morning, the average delays of A-B, A-C, A-E, AD, B-C, B-D, C-D, C-E and D-E are $50 \mathrm{~ms}, 300 \mathrm{~ms}, 30 \mathrm{~ms}, 5000 \mathrm{~ms}, 30 \mathrm{~ms}, 100 \mathrm{~ms}$, $50 \mathrm{~ms}, 30 \mathrm{~ms}$ and $15 \mathrm{~ms}$ respectively

To sum up, during the periods of 06:00 in the morning, 12:00 at noon and 18:00 in the afternoon, the net is in stable period while during the periods of 00:00 and 24:00 in the morning, the net is in peak period.

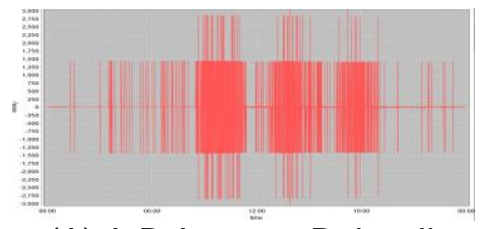

(A) A-B Averäge Delay Jitter

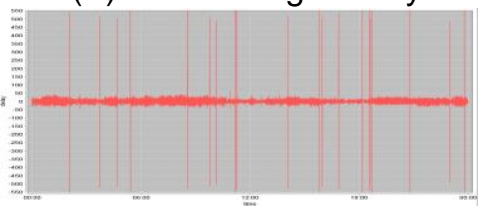

A-E Average Delay Jitter

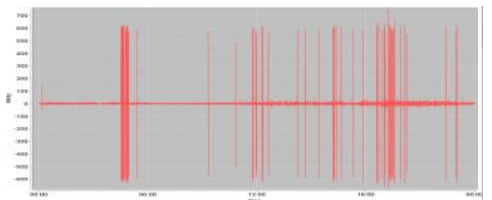

(E) B-C Average Delay Jitter

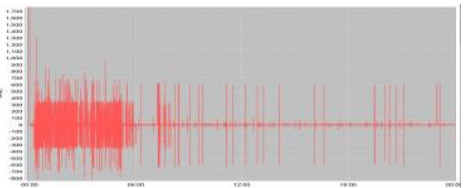

(G) C-D Average Delay Jitter

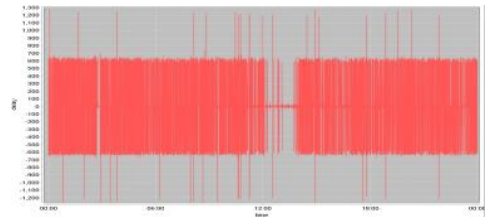

(B) A-C Average Delay Jitter

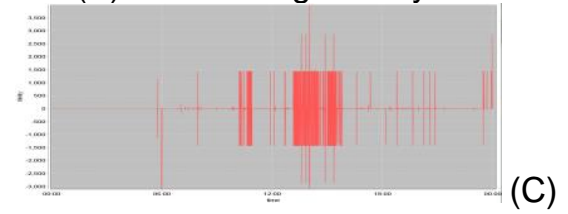

(D) A-D Average Delay Jitter

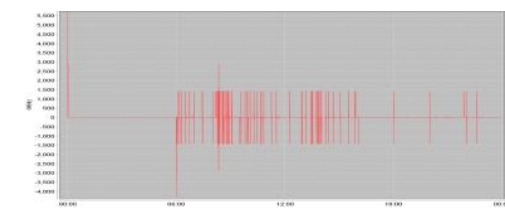

(F) B-D Average Delay Jitter

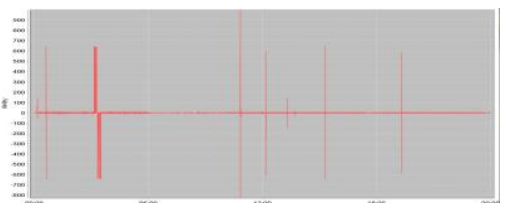

(H) C-E Average Delay Jitter

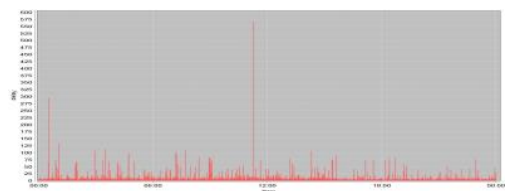

(I) D-E Average Delay Jitter

\section{Diagram 5: Daily Average Delay Jitter}

Diagram 5 (a) - (i) discusses the corresponding daily average delay jitter. The abscissa shows peak period, idle period and stable period, while the ordinate delay jitter (ms). During the period of 00:00 in the morning, the average delay jitters of A-B, A-C, A-E, A-D, B-C, B-D, C-D, C-E and D-E are respectively $[-30,30] \mathrm{ms}$, [$600,600] \mathrm{ms}, \quad[-30,30] \mathrm{ms}, \quad[-10,10] \mathrm{ms}, \quad[-100,100] \mathrm{ms}, \quad[-5000, \quad 5000] \mathrm{ms}, \quad[-$ $1500,1500] \mathrm{ms},[-150,150] \mathrm{ms},[-15,15] \mathrm{ms}$. During the period of 06:00 in the morning, the average delay jitters of A-B, A-C, A-E, A-D, B-C, B-D, C-D, C-E and D-E are respectively $[-1000,1000] \mathrm{ms},[-700,700] \mathrm{ms},[-40,40] \mathrm{ms},[-5000,5000] \mathrm{ms},[-30,30] \mathrm{ms}$, $[-5000,5000] \mathrm{ms},[-100,100] \mathrm{ms},[-20,20] \mathrm{ms},[-20,20] \mathrm{ms}$. During the period of 12:00 at noon, the average delay jitters of A-B, A-C, A-E, A-D, B-C, B-D, C-D, C-E and D-E are respectively $[-30,30] \mathrm{ms},[-50,50] \mathrm{ms},[-30,30] \mathrm{ms},[-1000,1000] \mathrm{ms},[-500,500] \mathrm{ms}$, $[-30,30] \mathrm{ms},[-30,30] \mathrm{ms},[-20,20] \mathrm{ms},[-25,25] \mathrm{ms}$. During the period of 18:00 in the afternoon, the average delay jitters of A-B, A-C, A-E, A-D, B-C, B-D, C-D, C-E and D-E are respectively $[-1000,1000] \mathrm{ms},[-600,600] \mathrm{ms},[-30,30] \mathrm{ms},[-10,10] \mathrm{ms},[-$ 
$80,80] \mathrm{ms},[-1500,1500] \mathrm{ms},[-50,50] \mathrm{ms},[-30,30] \mathrm{ms},[-20,20] \mathrm{ms}$. During the period of 24:00 in the morning, the average delay jitters of A-B, A-C, A-E, A-D, B-C, B-D, C$\mathrm{D}, \mathrm{C}-\mathrm{E}$ and D-E are respectively $[-30,30] \mathrm{ms},[-700,700] \mathrm{ms},[-30,30] \mathrm{ms}$, [$2000,2000] \mathrm{ms},[-50,50] \mathrm{ms},[-30,30] \mathrm{ms},[-50,50] \mathrm{ms},[-20,20] \mathrm{ms},[-40,40] \mathrm{ms}$.

To sum up, during the period of 12:00 at noon and 24:00 in the morning, the net is in stable period while during the period of 00:00 and 06:00 in the morning and 18:00 in the afternoon, the net is in peak period.

\section{GCTP Modeling and Application Analysis Based On EPSN}

\subsection{GCTP Modeling Based On EPSN}

In GCTP, every command can be regarded as multi-constraints multi-objective optimization problem (MCMOOP) and so GCTP can be seen as group multiconstraints multi-objective optimization problem (GMCMOOP) .

MCMOOP modeling: With every sub-object function satisfying metric constraints, look for a group of non-inferior solution to optimize the present MCMOOP object function (without loss of generality and with the definition of maximum as optimization):

$$
\begin{gathered}
\operatorname{Maxf}(x)=\left\{f_{1}(x), f_{2}(x), \ldots, f_{n}(x)\right\} \\
\text { s.t. }\left\{\begin{array}{l}
f_{j}(x) \leq C_{j}, j=1,2, \ldots \\
x \in E P
\end{array}\right.
\end{gathered}
$$

Wherein, $f_{j}$ represents the $j^{\text {th }}$ sub-object function of MCMOOP while $f$ the present MCMOOP object function. $C_{j}$ represents the restraints of $j^{\text {th }}$ object function of MCMOOP, $\mathrm{x}=\left\{\mathrm{x}_{1}, \mathrm{x}_{2}, \ldots, \mathrm{x}_{\mathrm{m}}\right\}$ represents $\mathrm{n}$ dimensional solution variables, while $\mathrm{EP}$ effective path set based on measurement and statistics.

GMCMOOP modeling: Look for multi-groups of non-inferior solution $\mathrm{GEP}_{1}$, $\mathrm{GEP}_{2}, \ldots, \mathrm{GEP}_{\mathrm{n}}$, with the $\mathrm{MCMOOP}_{\mathrm{j}}$ corresponding to any of $\mathrm{GEP}_{\mathrm{j}} \mathrm{j}=1,2, \ldots$ optimized, make the present GMCMOOP optimal.

$$
\begin{gathered}
M a x F=\left\{\operatorname{Max}_{1}, M a x F_{2}, \ldots, M a x F_{n}\right\} \\
\text { s.t. } \forall i, j, G E P_{i}, G E P_{j} \subset E P, i, j=1,2, \ldots
\end{gathered}
$$

Wherein, $F_{j}$ represents the $j^{\text {th }}$ MCMOOP while $F$ the present GMCMOOP object functions. $\mathrm{GEP}_{\mathrm{i}}, \mathrm{GEP}_{\mathrm{j}}$ respectively represents the non-inferior solution set of MCMOOP and $\mathrm{MCMOOP}_{\mathrm{j}}$ while EP effective path set based on measurement and statistics.

\subsection{Application Analysis}

Suppose in diagram 2 net structure, A transmits two commands $\mathrm{Com}_{1}$ and $\mathrm{Com}_{2}$ to $\mathrm{C}$ and $\mathrm{D}$ respectively, and the corresponding restraints are delay $100 \mathrm{~ms}$ with delay jitter of $100 \mathrm{~ms}$ and delay $90 \mathrm{~ms}$ with delay jitter of $90 \mathrm{~ms}$. Thus the operation center needs to calculate the effective path to enable $\mathrm{Com}_{1}$ and $\mathrm{Com}_{2}$ to achieve the goal while respectively satisfying the restraints.

Calculating and analyzing course:

Step 1 First construct EP set according to the measuring and statistical result of the daily average delay and daily average delay jitter in chapter 2.4.

Step 2 Construct the calculating model according to formulas (1) and (2). 
Step 3 Respectively calculate the effective path of $\mathrm{Com}_{1}$ and $\mathrm{Com}_{2}$ by means of basic particle swarm according to EP (EP-PSO) set and formulas (1) and (2).

Step 4 Respectively choose optimal effective path based on the calculating result.

Step 5 Go back to the result, save and log out.

Finally, the calculating and analyzing result is shown in diagram 6. Based on the calculating result, $\mathrm{Com}_{1}$ reaches $\mathrm{C}$ from $\mathrm{A}$ via the effective path $\mathrm{A}->\mathrm{B}-\mathrm{C}$ while $\mathrm{Com}_{2}$ reaches D from A via the effective path $\mathrm{A}->\mathrm{E}->\mathrm{D}$. (The red path represents $\mathrm{Com}_{1}$ while black for $\mathrm{Com}_{2}$ )

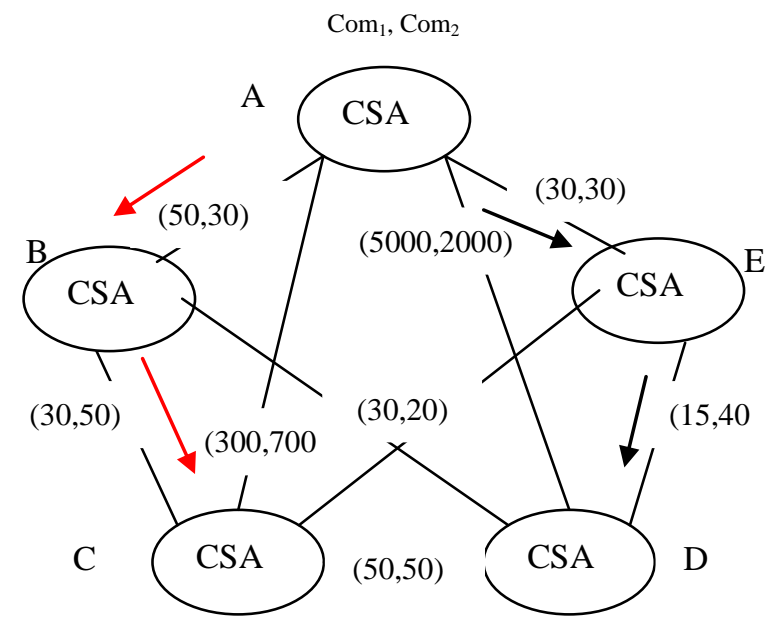

\section{Diagram 6: Calculating and Analyzing Result}

\section{Experiments and Analysis}

CSA environment configuration: 20 servers in dawning cluster with each having AMD Opteron 4122 for CPU. The memory is $4 \mathrm{G}$. The hard disk is $300 \mathrm{G}$ while the operation system is SUSE Linux Enterprise Server 11.

Net topology: refer to EPSN construction course in chapter 2.2 of this paper.

Selective course of EP set: refer to EPSN working thought of chapter 2.3 of this paper. This experiment randomly chooses as experiment data the EP set constructed on the basis of measuring and statistical result within a period of time.

Group command size: It is discovered from the measuring and statistical work that group command size has the minimum and maximum value of 50 and 100 respectively, which are chosen for the group command size in the experiment,

Performance test:

1) Verify the performance comparison of transmission success (TS) of particle swarm EP-PSO and basic particle swarm PSO based on EP set with EPSN scale constantly changing. $\mathrm{TS}=$ transmission success $/$ transmission total 


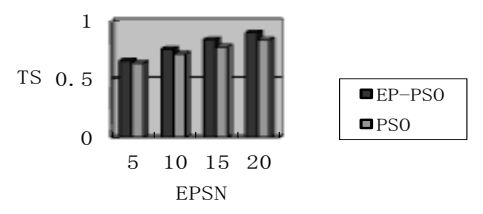

\section{Diagram 7: TS Comparison Under 50}

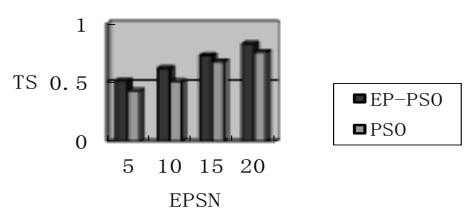

\section{Diagram 8: TS Comparison Under 100}

Diagram 7, the abscissa represents EPSN size, while the ordinate transmission success. When the EPSN size is 5 10, the EP-PSO transmission success is almost $71 \%$, and PSO calculation transmission success nearly 68\%. Compared with PSO calculation, EP-PSO transmission success has increased $4.41 \%$. When the EPSN size is 15 20, the EP-PSO transmission success is almost 87\%, and PSO calculation transmission success nearly $81 \%$. Compared with PSO calculation, EP-PSO transmission success has increased $7.4 \%$.

Diagram 8, the abscissa represents EPSN size, while the ordinate transmission success. When the EPSN size is 5 10, the EP-PSO transmission success is almost $56.65 \%$, and PSO calculation transmission success is nearly $46.6 \%$. Compared with PSO calculation, EP-PSO transmission success has increased $21.5 \%$. When the EPSN size is 15 20, the EP-PSO transmission success is almost 77.6\%, and PSO calculation transmission success is nearly $71.3 \%$. Compared with PSO calculation, EP-PSO transmission success has increased $8.83 \%$.

2) Verify the delay rate (DR) of particle swarm EP-PSO and basic particle swarm PSO based on EP with EPSN scale constantly changing. DR= delay time / normal time

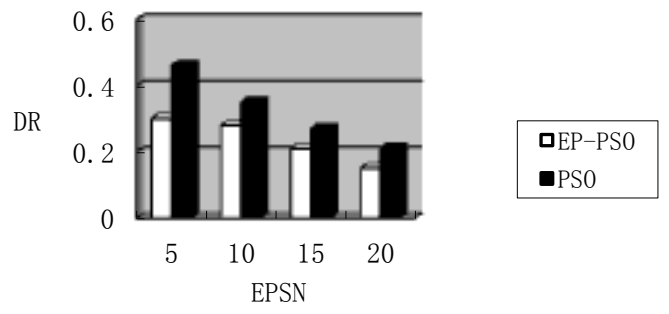

\section{Diagram 9: Delay Rate}

Diagram 9, the abscissa represents EPSN size, while the ordinate DR. When the EPSN size is 5 10, the EP-PSO delay rate is almost 29\%, and PSO calculation the delay rate is nearly $40.5 \%$. The delay rate has decreased $28.3 \%$. When the EPSN size 
is 15 20, the EP-PSO delay rate is almost $18 \%$, and PSO calculation delay rate is near $24 \%$. The delay rate has decreased $25 \%$ with the total decreasing $26.65 \%$.

To sum up, group command transmission course based on EP set can effectively increase group command transmission quality.

\section{Conclusion}

This paper presents effective path statistics network (EPSN) and converts group command transmission problem based on the Internet into one based on EPSN. It describes the definition, construction and working principle of EPSN and further samples and analyzes metrics according to EPSN. Based on the result the paper does modeling and calculating of group command transmission. The experiments show that group command transmission based on EPSN has better performance. But the paper doesn't consider the path competition among group commands, which is the focus of the next research work.

\section{Acknowledgements}

The authors gratefully acknowledge the support of Ministry of Education Doctoral Fund of PH.D supervisor of China (Grant No.20113120110008), Shanghai Key Science and Technology Project in Information Technology Field(No.14511107902), Shanghai Leading Academic Discipline Project(No. XTKX2012), Shanghai Engineering Research Center Project (No.GCZX14014, No. C14001).

\section{References}

[1] Luigi Atzori and Antonio Lera. From "Smart Objects" to "Social Objects". The Next Evolutionary Step of the Internet of Things[J]. IEEE Communications Magazine, 2014, 52(1):97-106.

[2] ZhengGuoSheng and ShuSenYang. A Survey on The IETF Protocol Suite for The Internet of Things:Standards, Challeges,and Opportunities[J]. IEEE Wireless Communications, 2013, 20(6):9198.

[3] A COHEN, E.KORACH and M.LAST. A fuzzy-based path ordering algorithm for QoS routing in non-deterministic communication networks[J]. Fuzzy Sets and Systems, 2005,150:401-417.

[4] R GURIN, A ORDA. QoS-based routing in network with inaccurate information:theory and algorithms[J]. IEEE/ACM Trans on Networking,1999,7(3):350-364.

[5] SUN Q, LANGENDOEFER H. Efficient multicast routing for delay-sensitive applications[C]/Proceedings of the 2nd Workshop on Protocols for Multimedia Systems(PROM'95). Salzburg, Austria:[s.n.],1995:452-458.

[6] W.MATTHEW CARLYLE,R.KEVIN WOOD. Near-shortest and K-shortest simple paths[J]. NETWORKS, 2005,46(2):98-109.

[7] Gang Liu and Ramakrishnan, K.G. A*Prune: an algorithm for finding K shortest paths subject to multiple constraints. INFOCOM 2001. Twentieth Annual Joint Conference of the IEEE Computer and Communications Societies[C]. Proceeding. IEEE. 2001, volume.2:743-749.

[8] Janardhan R. Iyengar. Concurrent Multipath Transfer Using SCTP Multihoming Over Independent End-to-End Pahts.[J] IEEE/ACM TRANSACTIONS ON NETWORKING, VOL 14, NO.5:951-964.

[9] WAXMAN B M. Performance evaluation of multipoint routing algorithms [A]. Proceedings of the INFOCOM'93 Conference[C] 1993:980-986.

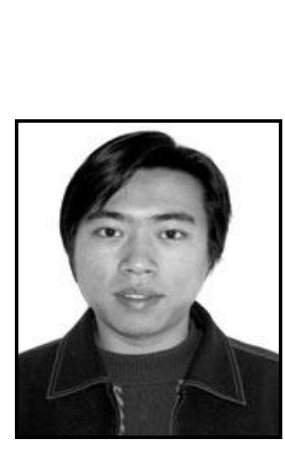

\section{Authors}

\section{Author's Name: Gang Zhang}

He's PHD candidate and interests in Internet of Things, computing network, QoS routing etc. 


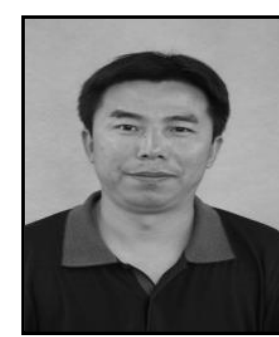

\section{Author's Name: Qing-Kui Chen}

He's PHD supervisor and interests in CSCW, parallel computing, computing network, QoS routing etc. 
International Journal of Hybrid Information Technology Vol.8, No.9 (2015) 